\title{
Acute Leukemia of Ambiguous Lineage with a Rare Abnormality Del17p by FISH Analysis
}

\author{
Naeem Abbas ${ }^{*}$, , Samra Waheed ${ }^{2}$, Aisha Jamal ${ }^{1}$, Ali Saleem ${ }^{1}$, Tahir Sultan Shamsi ${ }^{1}$ \\ ${ }^{1}$ Department of Clinical Hematology, National Institute of Blood Disease \& Bone Marrow Transplantation, Karachi, Pakistan. \\ ${ }^{2}$ Department of Hematology, National Institute of Blood Disease \& Bone Marrow Transplantation, Karachi, Pakistan.
}

\begin{abstract}
The World Health Organization (WHO) has categorized acute undifferentiated leukemia (AUL) as a rare subtype of acute leukemia of ambiguous lineage (ALAL). The prognosis of AUL is considered poor and it expresses no known lineage-specific markers. In majority of the cases, AUL has been associated with karyotypic abnormalities, most commonly deletion $5 \mathrm{q}$ and complex karyotype. Deletion $17 \mathrm{p}$ correlation with acute myeloid leukemia and myelodysplastic syndome has been previously established and is associated with poorer outcomes. Herein we are reporting a case of forty years old male who was referred to National institute of blood diseases and bone marrow transplantation with complains of fever, multiple neck swellings, and early satiety and was diagnosed as Acute Undifferentiated Leukemia along with deletion $17 \mathrm{p}$. This is a rare entity and can aid in further diagnostic and therapeutic approaches.
\end{abstract}

Keywords: Acute undifferentiated leukemia, Deletion 17p, Flourescnece in situ hybridization, Allogeneic haematopoetic stem cell transplantion, Flow cytometry.

\section{INTRODUCTION}

Diagnosis and classification of acute leukemia is based on morphology, immunophenotyping, chromosomal analysis and specific genetic tests [1]. In majority of cases diagnosis and lineage categorization in to myeloid, B-lymphoid or T-lymphoid can easily be established by multiparameter flowcytometry, but in few cases lineage attribution is problematic [2]. These cases which do not show lineage specific antigens are currently classified by WHO as acute undifferentiated leukemia which are the subtype of acute leukemia of ambiguous lineage [3].

Deletion of short arm of chromosome 17 (Del 17p) predicts shorter survival, resistance to available therapy in chronic lymphocytic leukemia, other non hodgkin's lymphoma and multiple myeloma cases $[4,5] .3-4 \%$ of acute myeloid leukemia and myelodysplastic syndrome cases exhibit this chromosomal abnormality [6]. We are reporting here a case of acute undifferentiated leukemia with del $17 \mathrm{p}$ chromosomal aberration.

\section{CASE PRESENTATION}

40 years old male patient was referred to National Institute of Blood Disease and Bone Marrow Transplantation with complains of multiple swellings in necks, intermittent fever and history of $5 \mathrm{~kg}$ weight loss in last 1 month. On examination both right and left cervical lymph nodes were palpable along with enlarge spleen that was palpable 2 fingers below the left costal margin. CBC showed Hemoglobin $10.7 \mathrm{G} / \mathrm{dl}$ Total Leukocyte Count $93.22 \times 10^{9} / \mathrm{L}$ and Platelets $60 \times 10^{9} / \mathrm{L}$

*Address correspondence to this author at the Department of Clinical Hematology, National Institute of Blood Disease \& Bone Marrow

Transplantation, Karachi, Pakistan. E-mail: naeemabbassolangi@gmail.com differential count showed 94\% Abnormal lymphoid cells as showed in (Fig. 1). These abnormal cells were small to medium in size further characterized by high nuclear to cytoplasmic ratio, scant pale blue agranular cytoplasm, condensed chromatin pattern and inconspicuous nucleoli as represented in (Fig. 2). No any myelodysplasia related findings were observed. The morphology of these cells was not straightforward indicator of acute nature of disease, so Del17p by FISH was also sent along with other diagnostic workup. Myeloperoxidase stain on aspirate smear was negative illustrated in (Fig. 3). Trephine section exhibited interstitial to diffuse infiltration by these abnormal cells as shown in (Fig. 4). Immunohistochemistry was performed for further evaluation and lineage attribution. Initial panel showed CD45 and TdT diffuse positivity as seen in (Fig. 5). While these cells did not express CD34 and CD117 represented in (Fig. 6). For lineage specification B-lymphoid (CD19, CD 79a, CD20) demonstrated in (Figs. 7, 8) T-lymphoid (CD3) shown in (Fig. 9). Myeloid (CD13, CD33) as seen in (Figs. 10, 11) these all markers were negative. Before concluding it as acute undifferentiated leukemia CD38 and CD56 exhibited in (Figs. 12, 13) were also performed to see for if plasmacytoid or NK cell precursors were present, but these blast cells did not express any lineage specific antigen. Immunophenotyping by Flowcytometry was also performed and diagnosis turned out to be same. Conventional cytogenetic analysis showed $46 \mathrm{XY}$ normal male karyotype, while del17p was detected by FISH analysis as illustrated in (Fig. $14)$. 


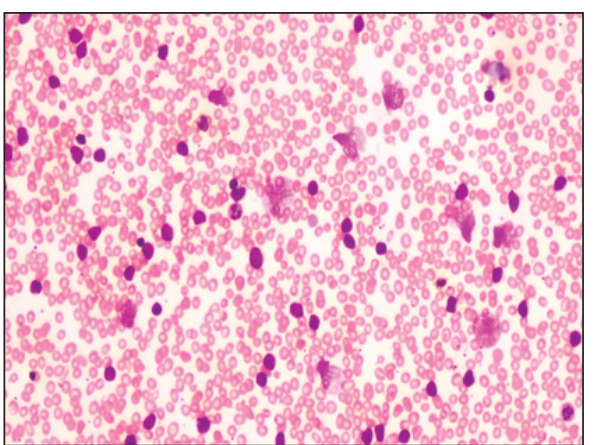

Fig. (1). Peripheral Smear of the Patient Exhibiting Blast Cells.

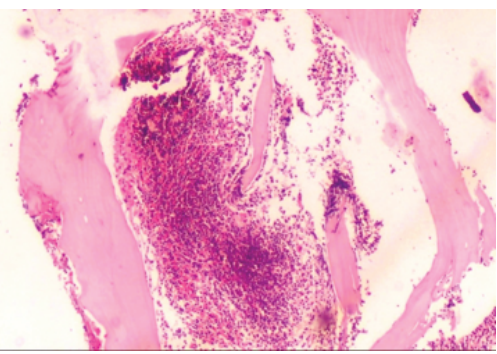

Fig. (4). Trephine Biopsy Showing Diffuse Infiltration by Blast Cells.

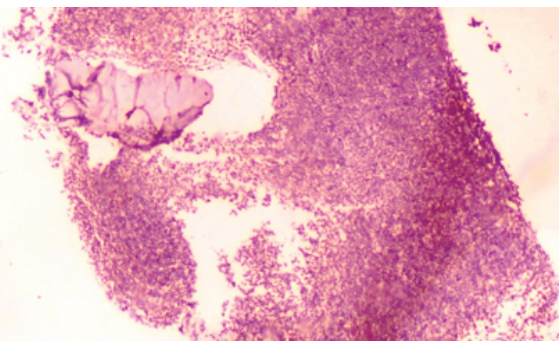

Fig. (7). Blast Population is CD19 Negative.

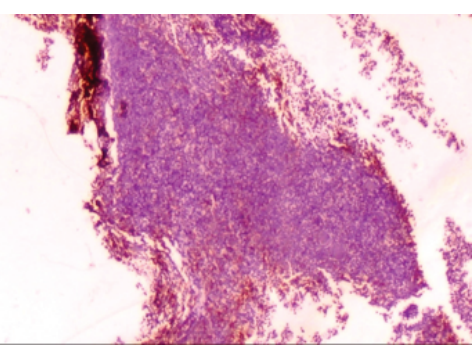

Fig. (10). Blast Cells Population is CD 13 Negative.

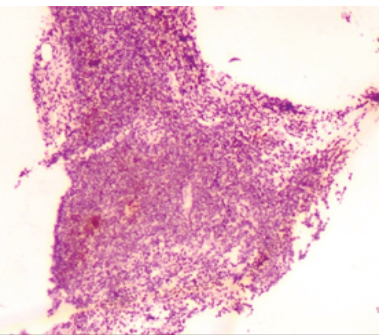

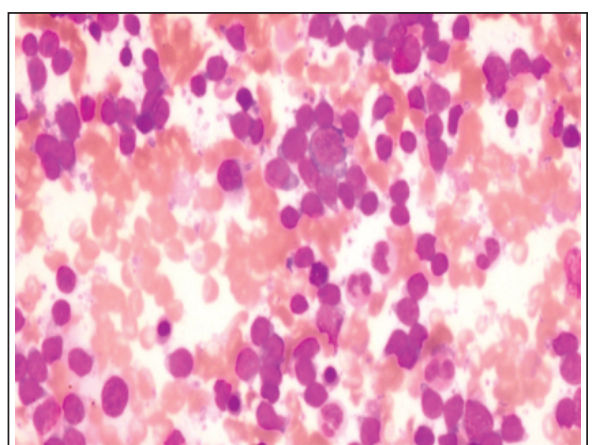

Fig. (2). Bone Marrow Aspirate

Showing Large Number of Blast Cells and Suppressed Trilineage

Hematopoiesis.

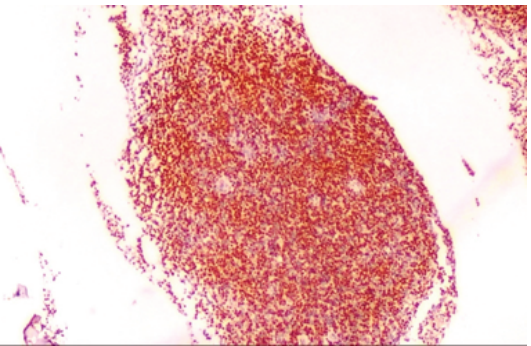

Fig. (5). Blast Population Showing Diffuse Positivity of TDT.

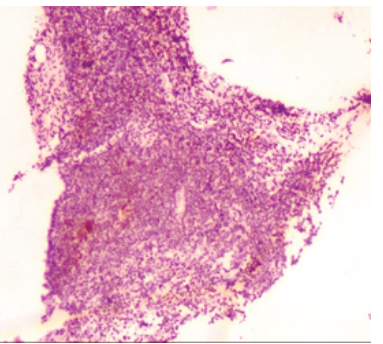

Fig. (8). Blast Cells Population is CD79a Negative.

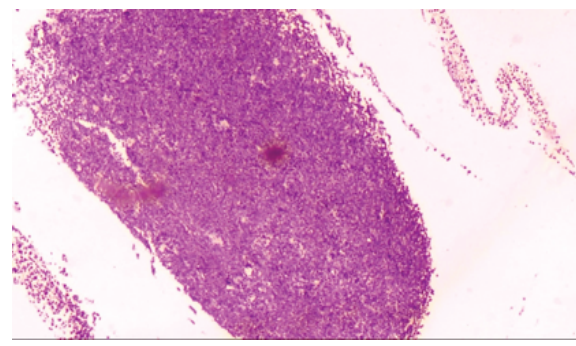

Fig. (11). Blast Cells Population is CD 33 Negative.

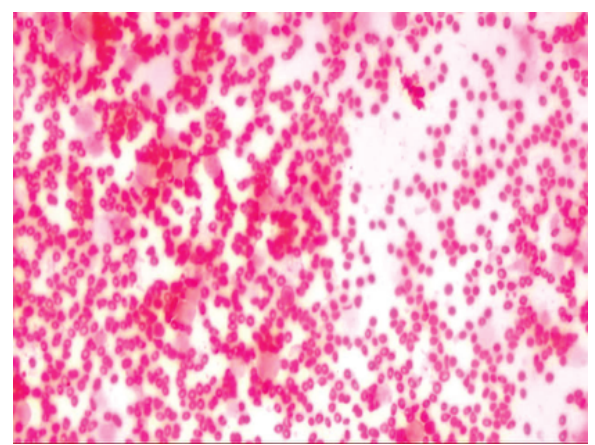

Fig. (3). Myeloperoxidase on Aspirate Smear Showing Blast Cells are MPO Negative.

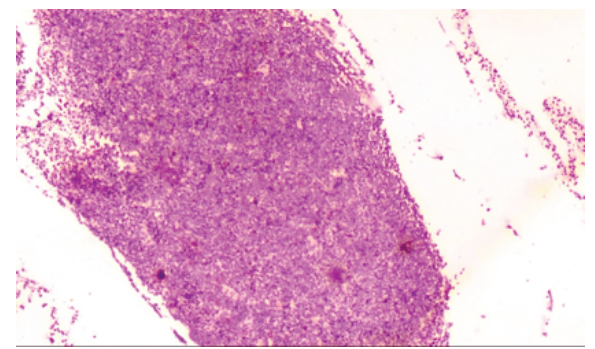

Fig. (6). Blast Population is CD117 Negative.

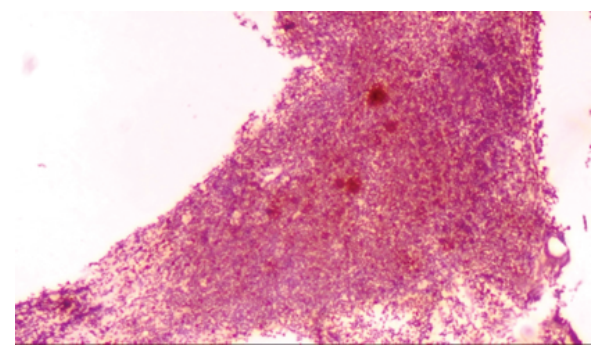

Fig. (9). Blast Cells Population is CD 3 Negative.

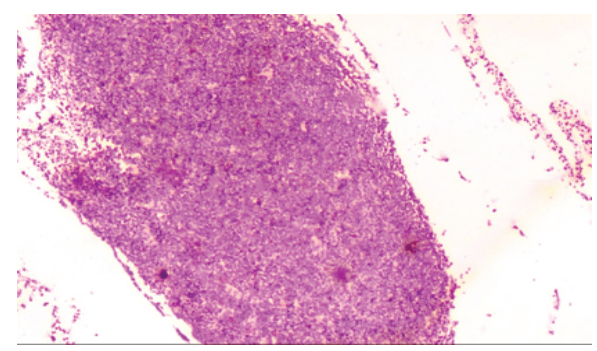

Fig. (12). Blast Cells Population is CD 38 Negative.

Fig. (13). Blast Cells Population is CD56 Negative. 


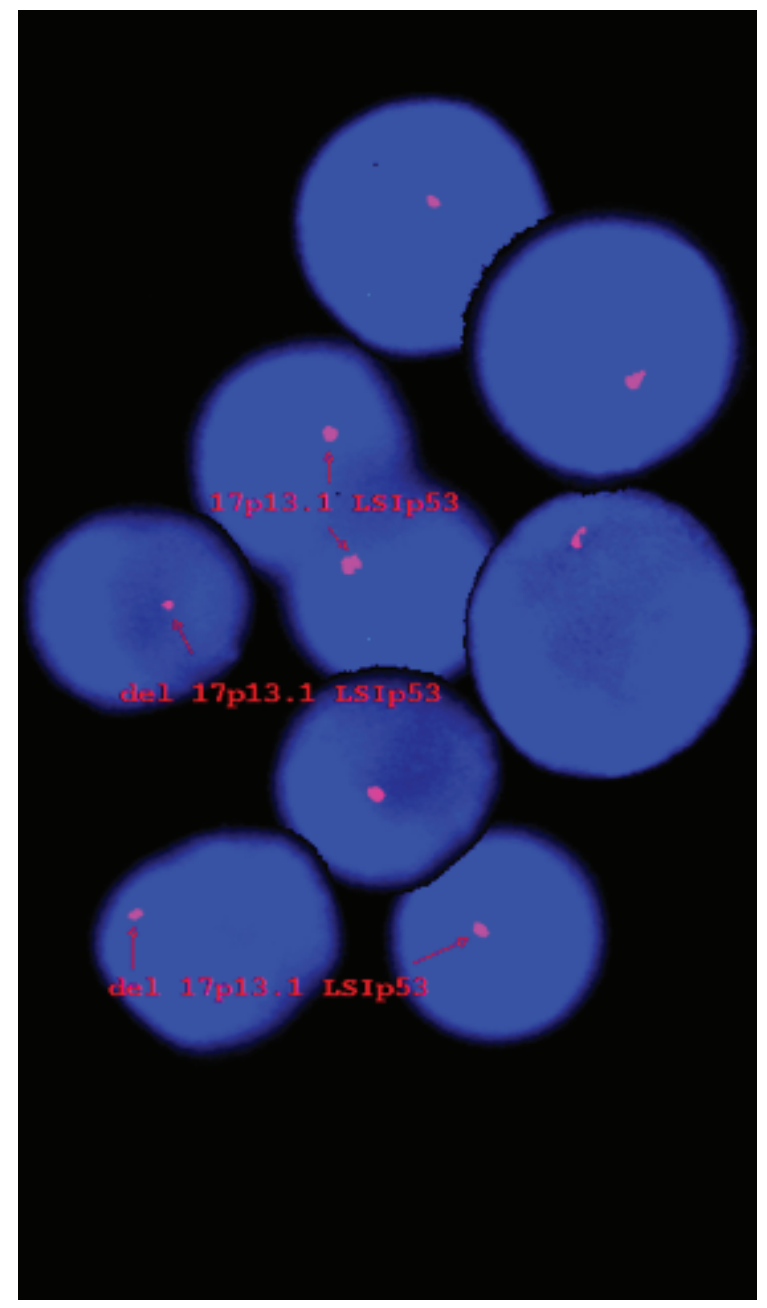

Fig. (14). Deletion 17p Analysis by FISH.

CT scan chest and abdomen with contrast showed extensive lymphadenopathy and hepatosplenomegaly. Echocardiography showed normal function of ventricles and viral markers were negative. Cytoreduction therapy with Hydroxyurea was started along with antiviral and antifungal prophylaxis, allopurinol was also started along with intravenous fluids to prevent tumor lysis syndrome, when cytoreduction was achieved by reduction of white blood cell count, he was offered with $(7+3)$ cytarabine and daunorubicin induction protocol. However, patient did not complete the treatment because of socioeconomic issues and was continued with low dose cytarabine according to the patient's will.

\section{DISCUSSION}

Acute Undifferentiated leukemia is more common in older adults with cytogenetic abnormalities in $80-90 \%$ of cases. Most frequent are del (5q) and trisomy 13 [7]. Cuneo et al. reported that del $(5 q)$ was seen in $33 \%$ of acute undifferentiated leukemia cases, trisomy of chromosome 13 in $33 \%$, and complex karyotype in only one case, Heesch et al. reported complex karyotype as a major cytogenetic abnormality $[2,8]$.

Del $17 p$ is associated with poor overall and disease free survival in Chronic Lymphoproliferative disease and multiple myeloma and is resistant to conventional chemotherapy regimens [5]. Its association in Acute Leukemia is sparsely reported and has been mostly associated with Acute Myeloid Leukemia and Myelodysplastic Syndrome. A large study of 2272 acute myeloid leukemia patients showed deletion $17 \mathrm{p}$ in 105 patients (05\%) [9]. The multivariate analysis exhibited del 17p (p53) aberrations as an independent negative prognostic factor for overall survival, disease-free survival and increased relapse risk.

Previously it has been reported in few studies that expression of Tdt is more frequently seen in acute undifferentiated leukemia rather than other markers of immaturity (CD34 and CD 117) mostly associated with Myeloid differentiation [10]. Our patient also had the same findings though no prognostic significance has yet been associated with this finding.

To the best of our knowledge and literature search, this is the first case of Acute Undifferentiated Leukemia associated with deletion $17 \mathrm{p}$, this could aid in including deletion $17 \mathrm{p}$ testing in Acute Leukemia especially those having aggressive or resistant disease.

\section{CONFLICT OF INTEREST}

Declared none.

\section{ACKNOWLEDGEMENTS}

We acknowledge all our fellow colleagues.

\section{AUTHORS' CONTRIBUTION}

All authors have been contributed equally.

\section{REFERENCES}

[1] Kurosawa S, Toya T, Kishida Y, et al. Outcome of patients with acute undifferentiated leukemia after allogeneic hematopoietic stem cell transplantation. Leuk Lymphoma 2018; 59(12): 3006-9. DOI: 10.1080/10428194.2018.1441410

[2] Foon KA, Schroff RW, Gale RP. Surface markers on leukemia and lymphoma cells: Recent advances. Blood 1982; 60(1): 1. DOI: 10.1182/blood.V60.1.1.bloodjournal6011

[3] Arber DA, Orazi A, Hasserjian R, et al. The 2016 revision to the World Health Organization classification of myeloid neoplasms and acute leukemia. Blood 2016; 127: 2391-405. DOI: 10.1182/blood-2016-03-643544

[4] Lode L, Eveillard M, Trichet V, et al. Mutations in TP53 are exclusively associated with del(17p) in multiple myeloma. Haematologica 2010; 95(11): 1973-6. DOI: 10.3324/haema- 
tol.2010.023697

[5] Zenz T, Vollmer D, Trbusek M, et al. TP53 mutation profile in chronic lymphocytic leukemia: Evidence for a disease specific profile from a comprehensive analysis of 268 mutations. Leukemia 2010; 24: 2072-9. DOI: 10.1038/leu.2010.208

[6] Lai' JL, Preudhomme C, Zandecki M, et al. Myelodysplastic syndromes and acute myeloid leukemia with $17 \mathrm{p}$ deletion. An entity characterized by specific dysgranulopoiesis and a high incidence of P53 mutations. Leukemia 1995; 9(3): 370-81. DOI: $10.1016 / 0145-2126(94) 90142-2$

[7] Cuneo A, Ferrant A, Michaux JL, et al. Cytogenetic and clinicobiological features of acute leukemia with stem cell phenotype: study of nine cases. Cancer Genet Cytogenet 1996; 92(1): 31-6. DOI: 10.1016/S0165-4608(96)00127-6
[8] Heesch S, Neumann M, Schwartz S, et al. Acute leukemias of ambiguous lineage in adults: Molecular and clinical characterization. Ann Hematol 2013; 92: 747-58.

DOI: $10.1007 / \mathrm{s} 00277-013-1694-4$

[9] Seifert H, Mohr B, Thiede C, et al. The prognostic impact of $17 \mathrm{p}$ (p53) deletion in 2272 adults with acute myeloid leukemia. Leukemia 2009; 23(4): 656-63

DOI: $10.1038 /$ leu.2008.375

[10] Olga K, Weinberg, Robert P, et al. Clinical, immunophenotypic, and genomic findings of acute undifferentiated leukemia and comparison to acute myeloid leukemia with minimal differentiation: A study from the bone marrow pathology group. Mod Pathol 2019; 32(9): 1373-85.

DOI: $10.1038 / \mathrm{s} 41379-019-0263-3$ 